\title{
Yokukansan and its ingredients as possible treatment options for schizophrenia
}

\author{
This article was published in the following Dove Press journal: \\ Neuropsychiatric Disease and Treatment \\ I September 2014 \\ Number of times this article has been viewed
}

\author{
Chuan-Hsun $\mathrm{Yu}^{\prime}$ \\ Ryouhei Ishii ${ }^{2}$ \\ Shun-Chieh Yu' \\ Masatoshi Takeda² \\ 'Yuli Hospital, Department \\ of Health, Hualien, Taiwan; \\ ${ }^{2}$ Department of Psychiatry, \\ Osaka University Graduate \\ School of Medicine, Osaka, Japan
}

Correspondence: Ryouhei Ishii

Department of Psychiatry, Osaka

University Graduate School of Medicine

2-2 D3, Yamadaoka, Suita City,

Osaka, Japan 565-087

Tel +81668793051

Fax+816 68793059

Email ishii@psy.med.osaka-u.ac.jp

\begin{abstract}
Schizophrenia is a debilitating psychotic mental disorder that affects almost the entire range of human mental function. The devastating effect of the illness is usually long-lasting and requires lifelong treatment. Despite an evolved psychopharmacological understanding, the overall therapeutic effect of antipsychotics is still not satisfactory. The choice of proper medication presents a clinical dilemma between efficacy and safety. As a result, searching for comparable treatment options with safer profiles is very important. Yokukansan (TJ-54), also called yi-gan san in Chinese, is a traditional herbal medicine with evident therapeutic effect for neuropsychiatric disorders. There are several open-label clinical studies upholding the possibility of using yokukansan to treat schizophrenia or schizophrenia-like psychosis. Evidence from animal studies and neurobiology also sheds light on the antipsychotic implications of yokukansan and its ingredients. Nevertheless, correlations between the experimental environment and clinical settings may be complicated by a number of confounders. Clinical trials with more sophisticated designs are required to fill the gap between the experimental environment and clinical settings.
\end{abstract}

Keywords: herbal medicine, geissoschizine methyl ether, glycyrrhizin, antipsychotics, D2 receptor

\section{Background}

Schizophrenia is often a severe and debilitating psychotic disorder. It usually begins in late adolescence or early adulthood and affects approximately $1 \%$ of the population. ${ }^{1}$ The devastating effect of the illness is usually long-lasting and requires lifelong treatment. Considering the substantial burden on patients, their families, and society, schizophrenia is becoming one of the most important public health problems. ${ }^{1}$ The core symptoms and signs of the illness include hallucinations, delusions, abnormal behaviors, and disorganized speech. Deficits in the ability to experience pleasure, flattening of emotional expression, loss of drive, and impoverishment of mental activity are also noticed. Vocational and social disabilities usually result from the accompanied cognitive impairments in memory, attention, and executive function. ${ }^{2}$ Almost the entire range of human mental function is affected by the disorder.

People with schizophrenia have difficulties in regulating information. They are unable to filter out the stimuli that most people can ignore $^{3}$ and lack the skills to assess their significance. ${ }^{4}$ Together with the paranoid psychopathology, patients become hypervigilant and struggle to decide what experiences are real. ${ }^{5}$ Violence in response to these symptoms may occur, ${ }^{6}$ as can suicidal behaviors. ${ }^{7}$ There are several pathological processes of the brain that express schizophrenia as a disease of the mind. The unfortunate convergence of these factors results in altered neurotransmitter mechanisms and loss of neuronal connectivity, both of which contribute to deficits in inhibitory function and the inability to sort information. ${ }^{5}$ 


\section{Antipsychotics}

Antipsychotics are the primary treatments for schizophrenia. Since the first discovery of the neuroleptic effects of chlorpromazine in the 1950s, modulation of dopamine D2 receptors has played an important role in treating psychotic disorders. ${ }^{8}$ Along with the similar modulation, newer-generation antipsychotics have been developed since the enhanced treatment effect of clozapine was reported in 1988. ${ }^{9}$ The pharmacological picture has also evolved, from understanding of simple blockade of D2 receptors to combined dopamine-serotonin antagonism, to even more advanced functionally selective actions at dopamine receptors. ${ }^{10-12}$ Nonetheless, the overall therapeutic effect is not satisfactory. Around one-fifth to one-third of patients with schizophrenia do not respond adequately to typical antipsychotics. ${ }^{13}$ The Clinical Antipsychotic Trials of Intervention Effectiveness (CATIE) showed that none of the examined medications could provide the majority of patients with a treatment they were able to tolerate for the full study period. ${ }^{14,15}$ Although olanzapine was found to be slightly superior to the conventional and other novel agents in terms of the Positive and Negative Syndrome Scale (PANSS) ratings, ${ }^{14}$ and clozapine was found to diminish the frequency of suicidal behavior, including serious suicidal acts, ${ }^{15}$ both clozapine and olanzapine are more likely to cause severe weight gain and potentially fatal metabolic problems. ${ }^{16,17}$ Patients with schizophrenia not only exhibit higher prevalence of metabolic syndrome, but are also at greater risk for developing cardiovascular mortality than the general population. ${ }^{18,19}$ Choosing proper medication is always a clinical dilemma. As a result, searching for comparable treatment options with safer profiles is very important.

\section{Yokukansan}

Traditional herbal medicines have drawn a lot of attention in recent decades regarding the balance between effectiveness and side effects. ${ }^{20-24}$ They have been utilized for a long time in the People's Republic of China and oriental countries, Japan, Taiwan, Korea and Vietnam. Herbal remedies were first introduced into Japan as Kampo medicine in the $5^{\text {th }}$ century and have been modified over hundreds of years in Japan. ${ }^{25}$ Yokukansan (TJ-54), also called yi-gan san in Chinese, is one of the Kampo prescriptions and has evident therapeutic effects for neuropsychiatric disorders. It contains a mixture of seven dried herbs, including Angelica acutiloba L. (Umbelliferae), Atractylodes lancea DC. (Compositae), Bupleurum falcatum L. (Umbelliferae), Poria cocos Wolf. (Polyporaceae), Glycyrrhiza uralensis (Leguminosae), Cnidium officinale Makino (Umbelliferae), and Uncaria rhynchophylla Schreb. (Rubiaceae) in a ratio of 3:4:2:4:1.5:3:3, respectively $^{26}$ (Table 1).
Table I Components of yokukansan

\begin{tabular}{ll}
\hline Constituent herb & Ratio of constituent* \\
\hline Angelica acutiloba L. (roots) & 3 \\
Atractylodes lancea DC. (roots) & 4 \\
Bupleurum falcatum L. (roots) & 2 \\
Poria cocos Wolf. (fungus) & 4 \\
Glycyrrhiza uralensis (roots) & $\mathrm{I} .5$ \\
Cnidium officinale Makino (roots) & 3 \\
Uncaria rhynchophylla Schreb. (hooks) & 3 \\
\hline
\end{tabular}

Note: *These are the ratios relative to each other.

The mixed extract is sold in packages with $2.5 \mathrm{~g}$ per unit and is usually taken three times daily before meals. ${ }^{25}$ Yokukansan was developed in 1555 by Xue Kai as a treatment for agitation and restlessness in children, ${ }^{20}$ and has been used in Japan for indications of insomnia, neurosis, irritability in children, and behavioral and psychological symptoms of dementia. ${ }^{22,27-30}$ Because of accumulating reports of yokukansan in treating psychiatric disorders, ${ }^{31}$ there is an emphasis on establishing its clinical implication in schizophrenia and schizophrenialike psychotic symptoms (Table 2).

Following the observation that yokukansan may have beneficial effects on dyskinesia, ${ }^{32}$ Miyaoka et al performed a 12-week, open-label study examining its efficacy, safety, and tolerability as an adjunctive treatment in 22 schizophrenic patients with tardive dyskinesia (TD). ${ }^{33}$ They found statistical improvement not only of TD, but also of psychotic symptoms. Transient nausea and constipation were reported in only two cases. However, after yokukansan was stopped, the condition of TD deteriorated. ${ }^{33}$ In order to evaluate its antipsychotic effects, the same group performed a 4-week, open-label control study to assess the usefulness of adding yokukansan in treating refractory patients with schizophrenia. ${ }^{34}$ A significant decrease was observed in each subscale in PANSS ratings by the end of 4 weeks. Mild nausea or tiredness were noticed in three cases, but no serious adverse events were reported. This result suggests that an adjunction of yokukansan might be effective for treatment-resistant schizophrenia. ${ }^{34}$ Furthermore, based on an experience of successful treatment of visual hallucination due to vision loss, ${ }^{35}$ a 4 -week, open-label study was initiated to investigate the monotherapeutic effect of yokukansan in treating Charles Bonnet Syndrome (CBS) ${ }^{36}$ A significant decrease of visual hallucination was revealed by psychometrics, including the Neuropsychiatric Inventory (NPI), the hallucination subscale of the PANSS, and Clinical Global Impression (CGI). CBS is defined by the triad of ocular pathology causing visual deterioration, complex visual hallucinations, and preserved cognitive status. The hypothetical pathophysiology is the deafferentation theory, which means a loss of sensory input after damage to neurons 
Table 2 Clinical trials using yokukansan for patients with schizophrenia and schizophrenia-like psychosis

\begin{tabular}{|c|c|c|c|c|}
\hline $\begin{array}{l}\text { Study, } \\
\text { year }\end{array}$ & $\begin{array}{l}\text { Sample size and } \\
\text { study design }\end{array}$ & $\begin{array}{l}\text { Average daily } \\
\text { dosage (g/day) mean } \pm \\
\text { standard deviation }\end{array}$ & Assessment & Adverse events \\
\hline Miyaoka, ${ }^{33}$ & 13 males & 7.5 & AIMS $(12.1 \pm 2.2->1.6 \pm 1.3)^{* *}$ & Nausea and \\
\hline \multirow[t]{4}{*}{2008} & 9 females & & PANSS: Positive $(22.2 \pm 4.2->16.6 \pm 3.1)^{* *}$ & constipation ( 2 cases) \\
\hline & Mean age: 57.1 years & & Negative $(26.5 \pm 6.3->\mid 4.5 \pm 2.3)^{* *}$ & \\
\hline & I2-week open-label study & & General $(42.9 \pm 6.7->25.0 \pm 4.9)^{* *}$ & \\
\hline & Adjunctive therapy & & CGI-S $(4.86 \pm 0.9->1.73 \pm 0.6)^{* *}$ & \\
\hline Miyaoka, ${ }^{34}$ & Treatment group & $6.7 \pm 2.5$ & PANSS: Positive $(27.7 \pm 6.1->11.9 \pm 3.7)^{* *}$ & Nausea ( 2 cases) \\
\hline \multirow[t]{9}{*}{2009} & 16 males & & Negative $(30.4 \pm 5.8->18.2 \pm 2.2) * *$ & Tiredness (I case) \\
\hline & 18 females & & General $(65 . I \pm 5.4->39.6 \pm 6.9)^{* *}$ & \\
\hline & Mean age: 45.5 years & & DIEPSS $(7.8 \pm 4.7->6.9 \pm 4.1)$ & \\
\hline & Control group & & & \\
\hline & 10 males & & & \\
\hline & 15 females & & & \\
\hline & Mean age: 45.2 years & & & \\
\hline & 4-week open-label control study & & & \\
\hline & Adjunctive therapy & & & \\
\hline Miyaoka, ${ }^{35}$ & A 2-week-treatment case report & 7.5 & Self-report & No adverse events \\
\hline 2009 & Monotherapy & & & reported \\
\hline Miyaoka, ${ }^{36}$ & 7 males & $5.8 \pm 2.6$ & NPI $(20.4 \pm 2.4->5.4 \pm I .5)^{* *}$ & Nausea (3 cases) \\
\hline \multirow[t]{4}{*}{2011} & 13 females & & HS-PANSS $(6.4 \pm 2.4->1.6 \pm 1.2)^{* *}$ & \\
\hline & Mean age: 66.3 years & & CGI $(4.5 \pm 1.8->1.3 \pm 0.3)^{* *}$ & \\
\hline & 4-week open-label study & & & \\
\hline & Monotherapy & & & \\
\hline Miyaoka, ${ }^{38}$ & 20 males & Baseline & BPRS $(36.7 \pm 4.65->20.1 \pm 1.6)^{*}$ & Tremor ( 3 cases) \\
\hline \multirow[t]{6}{*}{2013} & 20 females & $5.02 \pm 1.06$ & CGI-S $(5.03 \pm 0.89->1.73 \pm 0.55)^{*}$ & \\
\hline & Mean age: 73.1 years & Week 4 & PANSS $(66.0 \pm 7.3->34.3 \pm 2.2)^{*}$ & \\
\hline & 4-week open-label study & $5.23 \pm 1.22$ & Simpson-Angus Scale $(0.27 \pm 0.45->0.15 \pm 0.36)$ & \\
\hline & Monotherapy & & Barnes Akathisia Rating Scale & \\
\hline & & & $(0.20 \pm 0.4 I->0.13 \pm 0.34)$ & \\
\hline & & & AIMS $(0.23 \pm 0.42->0.18 \pm 0.39)$ & \\
\hline
\end{tabular}

Notes: $* p<0.001, * * p<0.0001$.

Abbreviations: AIMS, Abnormal Involuntary Movement Scale; BPRS, Brief Psychiatric Rating Scale; CGI, Clinical Global Impression; CGI-S, CGI-Severity; DIEPSS, DrugInduced Extrapyramidal Symptom Scale; HS-PANSS, hallucination subscale of the PANSS; NPI, Neuropsychiatric Inventory; PANSS, Positive and Negative Symptom Scale.

leading to excessive response of the deafferented neurons. ${ }^{37}$ In the central nervous system, this results in cortical hyperexcitability, a similar phenomenon observed in mechanisms of schizophrenia-like psychosis..$^{5}$ There is no established treatment for CBS. The positive result of these trials support Yokukansan as possible treatment for psychosis.

Recently, a more naturalistic 4-week, open-label study of yokukansan monotherapy for very-late-onset schizophrenia was conducted. ${ }^{38}$ Considerable improvement of psychotic symptomatology was noticed in all 40 patients. The statistically significant reduction on psychopathology was shown in all measures, including the Brief Psychiatric Rating Scale (BPRS), CGI-Severity, and the PANSS. Scores on the Simpson-Angus Scale, Barnes Akathisia Rating Scale, and Abnormal Involuntary Movement Scale (AIMS) decreased slightly but not significantly. There was no impairment in overall cognitive function as measured by the Mini-Mental State Examination (MMSE). These preliminary results demonstrate the possibility that yokukansan could be an effective and safe treatment option for schizophrenia.

Evidence from animal studies and neurobiology also sheds light on the antipsychotic implications of yokukansan. Several neurotransmitter systems involved in the neuropsychiatric effects of yokukansan and its ingredients have important roles in schizophrenia. Yokukansan was reported to inhibit the increased release of glutamate in zinc-deficient rats, which was regarded as a neurological disease model with the perturbed glutamatergic neurotransmitter system, and a modulation function on excitatory neurotransmitters was suggested. ${ }^{39}$ A further study revealed that yokukansan exerted a neuroprotective effect by ameliorating the dysfunction of glutamate transporters in astrocytes. ${ }^{40}$ This was mediated by one of the constituents of yokukansan, $G$. uralensis, and its main metabolite, $18 \beta$-glycyrrhetinic acid, which can access the brain through the blood-brain barrier. ${ }^{41,42}$ However, 
excessive release of glutamate not only induces neuronal death, as described in neurodegenerative disorders, ${ }^{43}$ but also enhances the frequency of excitatory postsynaptic potentials in cortical pyramidal cells, as observed in schizophrenia. . $^{54}$ These processes might be reversed by yokukansan in some way.

Repeated administration of yokukansan was found to decrease expression of 5-hydroxytryptamine $(5-\mathrm{HT})_{2 \mathrm{~A}}$ receptors in the prefrontal cortex and inhibit the head-twitch response induced by serotonin $5-\mathrm{HT}_{2 \mathrm{~A} / 2 \mathrm{C}}$ receptor agonist in mice. ${ }^{45}$ These results suggest the involvement of the serotonin system in the psychopharmacological effects of yokukansan. Constituent analysis of yokukansan revealed that compounds with affinities for $5-\mathrm{HT}_{1 \mathrm{~A}}, 5-\mathrm{HT}_{2 \mathrm{~A}}, 5-\mathrm{HT}_{2 \mathrm{C}}$, and 5- $\mathrm{HT}_{7}$ were only contained in U. rhynchophylla ${ }^{46}$ Further study demonstrated that geissoschizine methyl ether, a corynanthean-type alkaloid contained in U. rhynchophylla, had high blood-brain barrier permeability ${ }^{47}$ and behaved as a partial agonist at the serotonin $5-\mathrm{HT}_{1 \mathrm{~A}}$ receptor and as an antagonist at the serotonin $5-\mathrm{HT}_{2 \mathrm{~A}}, 5-\mathrm{HT}_{2 \mathrm{C}}$, and $5-\mathrm{HT}_{7}$ receptors. ${ }^{48}$

\section{Geissoschizine methyl ether}

Geissoschizine methyl ether is an indole alkaloid with a $\beta$-carboline structure, also called methyl (16Z,19E)-16(methoxymethylidene)coryn-19-en-17-oate (Figure 1). The molecular formula is $\mathrm{C}_{22} \mathrm{H}_{26} \mathrm{~N}_{2} \mathrm{O}_{3}$, and its molecular weight is 366.4534. $\beta$-carboline derivatives have been shown to have different affinities at different 5-HT receptors, depending on their ring substituents and ring saturation. ${ }^{49}$ An in vivo experiment also showed that geissoschizine methyl ether could ameliorate isolation-induced increased aggressiveness and decreased sociality, a function thought to be related

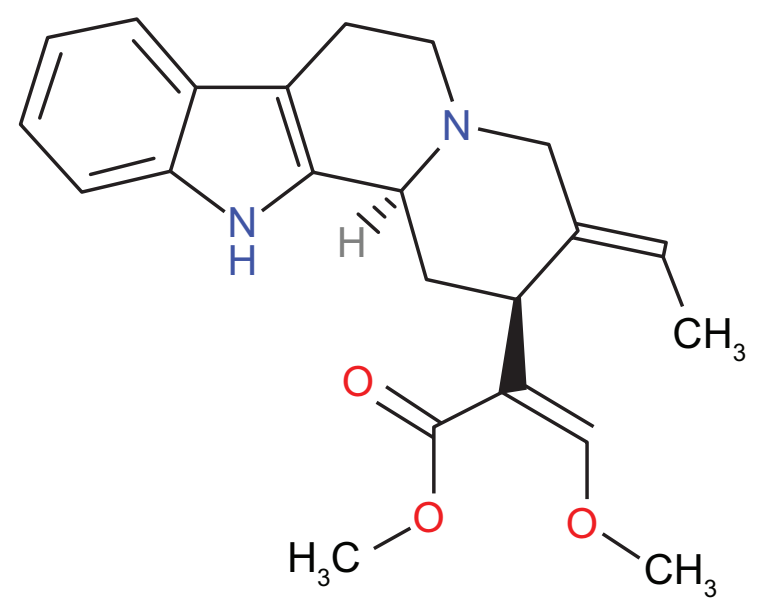

Figure I Chemical structure of geissoschizine methyl ether. Notes: Molecular formula: $\mathrm{C}_{22} \mathrm{H}_{26} \mathrm{~N}_{2} \mathrm{O}_{3}$. Molecular weight: $366.4534 \mathrm{~g} / \mathrm{mol}$. to $5-\mathrm{HT}_{1 \mathrm{~A}}$ agonist. $^{50}$ Atypical antipsychotics often exhibit serotonergic properties, especially $5-\mathrm{HT}_{2 \mathrm{~A}}$ and $5-\mathrm{HT}_{2 \mathrm{C}}$ receptor antagonism. ${ }^{10}$ Activation of the $5-\mathrm{HT}_{1 \mathrm{~A}}$ receptor has been suggested to decrease extrapyramidal symptoms and increase dopaminergic neurotransmission in the frontal cortex. ${ }^{51}$ Although the clear role for the $5-\mathrm{HT}_{7}$ receptor is not fully understood, blockade of it could be useful in treatment of psychotic symptoms, ${ }^{52}$ and some atypical antipsychotics, such as clozapine and risperidone, express high antagonistic affinity for this receptor.

Besides its serotonergic function, geissoschizine methyl ether has also been reported to be a partial agonist/antagonist at the cloned dopamine $\mathrm{D}_{2 \text { Long }}$ receptors with a low intrinsic activity and a partial activation response. ${ }^{48}$ All these qualities are similar to the properties found in aripiprazole. Aripiprazole, a so-called third-generation antipsychotic, acts on $\mathrm{D}_{2 \mathrm{Long}}{ }^{-}$ mediated signaling pathways with multiple intrinsic activities. This functionally selective profile, combined with actions at other neurotransmitter systems, was suggested to be responsible for the development of novel antipsychotics. ${ }^{11}$ Moreover, yokukansan has been proven to increase central acetylcholine release, ${ }^{53}$ and this atypical antipsychotic-like property may be helpful in preserving cognitive ability in schizophrenia. ${ }^{54}$

\section{Adverse events}

Some reversible adverse events of yokukansan have been reported, including sedation, ${ }^{22}$ vomiting/diarrhea, nausea, epigastric discomfort, leg edema, and hypokalemia. ${ }^{28}$ Glycyrrhizin, contained in G. uralensis, is able to facilitate potassium excretion in the renal tubules, and this may result in hypokalemia. ${ }^{28}$ There are no metabolic side effects documented. On the contrary, a large epidemiological study disclosed that the prevalence of diabetes was significantly increased for patients who received clozapine, olanzapine, and quetiapine. ${ }^{55}$ Nonetheless, Izumi et al found that yokukansan was able to suppress lipid synthesis and reduce fat accumulation in adipocytes by modulating the transcription factors without affecting glucose uptake. ${ }^{56}$ Considering the increased metabolic problems in patients with schizophrenia, choosing a drug without aggravation of lipid metabolism is essential in clinical practice.

\section{Conclusion}

This review of the literature highlights the possibility of yokukansan and its ingredients as new treatment options for schizophrenia. Nevertheless, correlations between the experimental environment and clinical settings may be complicated by a number of confounders. In order to bridge between basic 
research and clinical applications, further studies with more sophisticated designs are necessary.

\section{Acknowledgment}

This research was partially supported by the Center of Innovation Program from Japan Science and Technology Agency (JST).

\section{Disclosure}

The authors report no conflicts of interest in this work.

\section{References}

1. Andreasen NC. Understanding the causes of schizophrenia. $N$ Engl J Med. 1999;340(8):645-647.

2. Green MF. What are the functional consequences of neurocognitive deficits in schizophrenia? Am J Psychiatry. 1996;153(3):321-330.

3. Venables PH. Input dysfunction in schizophrenia. Prog Exp Pers Res. 1964;72:1-47.

4. Park S, Holzman PS, Goldman-Rakic PS. Spatial working memory deficits in the relatives of schizophrenic patients. Arch Gen Psychiatry. 1995;52(10):821-828.

5. Freedman R. Schizophrenia. N Engl J Med. 2003;349(18): $1738-1749$.

6. Swanson JW, Holzer CE 3rd, Ganju VK, Jono RT. Violence and psychiatric disorder in the community: evidence from the Epidemiologic Catchment Area surveys. Hosp Community Psychiatry. 1990;41(7): 761-770.

7. Siris SG. Suicide and schizophrenia. J Psychopharmacol. 2001;15(2): $127-135$.

8. Kapur S, Mamo D. Half a century of antipsychotics and still a central role for dopamine D2 receptors. Prog Neuropsychopharmacol Biol Psychiatry. 2003;27(7):1081-1090.

9. Kane J, Honigfeld G, Singer J, Meltzer H. Clozapine for the treatmentresistant schizophrenic. A double-blind comparison with chlorpromazine. Arch Gen Psychiatry. 1988;45(9):789-796.

10. Meltzer HY. Clinical studies on the mechanism of action of clozapine: the dopamine-serotonin hypothesis of schizophrenia. Psychopharmacology (Berl). 1989;99 Suppl:S18-S27.

11. Matsubara S, Meltzer HY. Effect of typical and atypical antipsychotic drugs on 5-HT 2 receptor density in rat cerebral cortex. Life Sci. 1989; 45(15):1397-1406.

12. Urban JD, Vargas GA, von Zastrow M, Mailman RB. Aripiprazole has functionally selective actions at dopamine D2 receptor-mediated signaling pathways. Neuropsychopharmacology. 2007;32(1): $67-77$.

13. Conley RR, Buchanan RW. Evaluation of treatment-resistant schizophrenia. Schizophr Bull. 1997;23(4):663-674.

14. Lieberman JA, Stroup TS, McEvoy JP, et al; Clinical Antipsychotic Trials of Intervention Effectiveness (CATIE) Investigators. Effectiveness of antipsychotic drugs in patients with chronic schizophrenia. N Engl J Med. 2005;353(12):1209-1223.

15. Modestin J, Dal Pian D, Agarwalla P. Clozapine diminishes suicidal behavior: a retrospective evaluation of clinical records. $J$ Clin Psychiatry. 2005;66(4):534-538.

16. Lindenmayer JP, Czobor P, Volavka J, et al. Changes in glucose and cholesterol levels in patients with schizophrenia treated with typical or atypical antipsychotics. Am J Psychiatry. 2003;160(2):290-296.

17. American Diabetes Association; American Psychiatric Association; American Association of Clinical Endocrinologists; North American Association for the Study of Obesity. Consensus development conference on antipsychotic drugs and obesity and diabetes. J Clin Psychiatry. 2004;65(2):267-272.
18. McEvoy JP, Meyer JM, Goff DC, et al. Prevalence of the metabolic syndrome in patients with schizophrenia: baseline results from the Clinical Antipsychotic Trials of Intervention Effectiveness (CATIE) schizophrenia trial and comparison with national estimates from NHANES III. Schizophr Res. 2005;80(1):19-32.

19. Saha S, Chant D, McGrath J. A systematic review of mortality in schizophrenia: is the differential mortality gap worsening over time? Arch Gen Psychiatry. 2007;64(10):1123-1131.

20. Aizawa R, Kanbayashi T, Saito Y, et al. Effects of Yoku-kan-san-kachimpi-hange on the sleep of normal healthy adult subjects. Psychiatry Clin Neurosci. 2002;56(3):303-304.

21. Iwasaki K, Kobayashi S, Chimura Y, et al. A randomized, double-blind, placebo-controlled clinical trial of the Chinese herbal medicine "ba wei di huang wan" in the treatment of dementia. J Am Geriatr Soc. 2004;52(9):1518-1521.

22. Iwasaki K, Satoh-Nakagawa T, Maruyama M, et al. A randomized, observer-blind, controlled trial of the traditional Chinese medicine YiGan San for improvement of behavioral and psychological symptoms and activities of daily living in dementia patients. J Clin Psychiatry. 2005;66(2):248-252.

23. Iwasaki K, Kato S, Monma Y, et al. A pilot study of banxia houpu tang, a traditional Chinese medicine, for reducing pneumonia risk in older adults with dementia. J Am Geriatr Soc. 2007;55(12):2035-2040.

24. Horiguchi J. [Clinical usage of Yi-gan san-schizophrenia, borderline personality disorder, dyskinesia etc]. Seishin Shinkeigaku Zasshi. 2012;114(6):708-718. Japanese.

25. Okamoto H, Okami T, Ikeda M, Takeuchi T. Effects of Yoku-kan-san on undifferentiated somatoform disorder with tinnitus. Eur Psychiatry. 2005;20(1):74-75.

26. de Caires S, Steenkamp V. Use of Yokukansan (TJ-54) in the treatment of neurological disorders: a review. Phytother Res. 2010;24(9): $1265-1270$.

27. Tateno M, Ukai W, Ono T, Saito S, Hashimoto E, Saito T. Neuroprotective effects of Yi-Gan San against beta amyloid-induced cytotoxicity on rat cortical neurons. Prog Neuropsychopharmacol Biol Psychiatry. 2008;32(7):1704-1707.

28. Mizukami K, Asada T, Kinoshita T, et al. A randomized cross-over study of a traditional Japanese medicine (kampo), yokukansan, in the treatment of the behavioural and psychological symptoms of dementia. Int J Neuropsychopharmacol. 2009;12(2):191-199.

29. Kung FC, Ishii R, Liu HC, Takeda M. New possibility of traditional Chinese and Japanese medicine as treatment for behavioral and psychiatric symptoms in dementia. Clin Interv Aging. 2012;7:393-396.

30. Mizukami K. Kampo therapy as an alternative to pharmacotherapy using antipsychotic medicines for behavioral and psychological symptoms of dementia (BPSD). Psychogeriatrics. 2008;8(3):137-141.

31. Miyaoka T, Horiguchi J. Clinical potential of Yi-gan san (Yokikansan) for psychiatric disorders. Curr Psychiatry Rev. 2009;5(4):271-275.

32. Furuya M, Kunishige K, J. H. [Three case of Lewy body disease with hallucination successfully treated with yi-gan san]. Seishin Igaku. 2007; 49:417-420. Japanese.

33. Miyaoka T, Furuya M, Yasuda H, et al. Yi-gan san for the treatment of neuroleptic-induced tardive dyskinesia: an open-label study. Prog Neuropsychopharmacol Biol Psychiatry. 2008;32(3):761-764.

34. Miyaoka T, Furuya M, Yasuda H, et al. Yi-gan san as adjunctive therapy for treatment-resistant schizophrenia: an open-label study. Clin Neuropharmacol. 2009;32(1):6-9.

35. Miyaoka T, Nagahama M, Tsuchie K, et al. Charles Bonnet syndrome: successful treatment of visual hallucinations due to vision loss with Yi-gan san. Prog Neuropsychopharmacol Biol Psychiatry. 2009;33(2): 382-383.

36. Miyaoka T, Furuya M, Kristian L, et al. Yi-gan san for treatment of charles bonnet syndrome (visual hallucination due to vision loss): an open-label study. Clin Neuropharmacol. 2011;34(1):24-27.

37. Plummer C, Kleinitz A, Vroomen P, Watts R. Of Roman chariots and goats in overcoats: the syndrome of Charles Bonnet. J Clin Neurosci. 2007;14(8):709-714. 
38. Miyaoka T, Wake R, Furuya M, et al. Yokukansan (TJ-54) for treatment of very-late-onset schizophrenia-like psychosis: an open-label study. Phytomedicine. 2013;20(7):654-658.

39. Takeda A, Itoh H, Tamano H, Yuzurihara M, Oku N. Suppressive effect of Yokukansan on excessive release of glutamate and aspartate in the hippocampus of zinc-deficient rats. Nutr Neurosci. 2008;11(1):41-46.

40. Kawakami Z, Kanno H, Ueki T, et al. Neuroprotective effects of yokukansan, a traditional Japanese medicine, on glutamate-mediated excitotoxicity in cultured cells. Neuroscience. 2009;159(4):1397-1407.

41. Kawakami Z, Ikarashi Y, Kase Y. Glycyrrhizin and its metabolite 18 beta-glycyrrhetinic acid in glycyrrhiza, a constituent herb of yokukansan, ameliorate thiamine deficiency-induced dysfunction of glutamate transport in cultured rat cortical astrocytes. Eur J Pharmacol. 2010;626(2-3):154-158.

42. Tabuchi M, Imamura S, Kawakami Z, Ikarashi Y, Kase Y. The bloodbrain barrier permeability of $18 \beta$-glycyrrhetinic acid, a major metabolite of glycyrrhizin in Glycyrrhiza root, a constituent of the traditional Japanese medicine yokukansan. Cell Mol Neurobiol. 2012;32(7): 1139-1146.

43. Mehta A, Prabhakar M, Kumar P, Deshmukh R, Sharma PL. Excitotoxicity: bridge to various triggers in neurodegenerative disorders. Eur J Pharmacol. 2013;698(1-3):6-18.

44. Aghajanian GK, Marek GJ. Serotonin model of schizophrenia: emerging role of glutamate mechanisms. Brain Res Brain Res Rev. 2000;31(2-3):302-312.

45. Egashira N, Iwasaki K, Ishibashi A, et al. Repeated administration of Yokukansan inhibits DOI-induced head-twitch response and decreases expression of 5-hydroxytryptamine (5-HT)2A receptors in the prefrontal cortex. Prog Neuropsychopharmacol Biol Psychiatry. 2008;32(6):1516-1520.

46. Terawaki K, Ikarashi Y, Sekiguchi K, Nakai Y, Kase Y. Partial agonistic effect of yokukansan on human recombinant serotonin 1A receptors expressed in the membranes of Chinese hamster ovary cells. J Ethnopharmacol. 2010;127(2):306-312.

47. Imamura S, Tabuchi $\mathrm{M}$, Kushida $\mathrm{H}$, et al. The blood-brain barrier permeability of geissoschizine methyl ether in Uncaria hook, a galenical constituent of the traditional Japanese medicine yokukansan. Cell Mol Neurobiol. 2011;31(5):787-793.
48. Ueda T, Ugawa S, Ishida Y, Shimada S. Geissoschizine methyl ether has third-generation antipsychotic-like actions at the dopamine and serotonin receptors. Eur J Pharmacol. 2011;671(1-3):79-86.

49. Glennon RA, Dukat M, Grella B, et al. Binding of beta-carbolines and related agents at serotonin (5-HT(2) and 5-HT(1A)), dopamine $(\mathrm{D}(2))$ and benzodiazepine receptors. Drug Alcohol Depend. 2000;60(2):121-132.

50. Nishi A, Yamaguchi T, Sekiguchi K, et al. Geissoschizine methyl ether, an alkaloid in Uncaria hook, is a potent serotonin 1A receptor agonist and candidate for amelioration of aggressiveness and sociality by yokukansan. Neuroscience. 2012;207:124-136.

51. Newman-Tancredi A. The importance of 5-HT1A receptor agonism in antipsychotic drug action: rationale and perspectives. Curr Opin Investig Drugs. 2010;11(7):802-812.

52. Galici R, Boggs JD, Miller KL, Bonaventure P, Atack JR. Effects of SB-269970, a 5-HT7 receptor antagonist, in mouse models predictive of antipsychotic-like activity. Behav Pharmacol. 2008;19(2):153-159.

53. Uchida N, Takasaki K, Sakata Y, et al. Cholinergic involvement and synaptic dynamin 1 expression in Yokukansan-mediated improvement of spatial memory in a rat model of early Alzheimer's disease. Phytother Res. 2013;27(7):966-972.

54. Ichikawa J, Dai J, O’Laughlin IA, Fowler WL, Meltzer HY. Atypical, but not typical, antipsychotic drugs increase cortical acetylcholine release without an effect in the nucleus accumbens or striatum. Neuropsychopharmacology. 2002;26(3):325-339.

55. Sernyak MJ, Leslie DL, Alarcon RD, Losonczy MF, Rosenheck R. Association of diabetes mellitus with use of atypical neuroleptics in the treatment of schizophrenia. Am J Psychiatry. 2002;159(4):561-566.

56. Izumi M, Seki T, Iwasaki K, Sakamoto K. Chinese herbal medicine Yi-Gan San decreases the lipid accumulation in mouse 3T3-L1 adipocytes by modulating the activities of transcription factors SREBP-1c and FoxO1. Tohoku J Exp Med. 2009;219(1):53-62.
Neuropsychiatric Disease and Treatment

\section{Publish your work in this journal}

Neuropsychiatric Disease and Treatment is an international, peerreviewed journal of clinical therapeutics and pharmacology focusing on concise rapid reporting of clinical or pre-clinical studies on a range of neuropsychiatric and neurological disorders. This journal is indexed on PubMed Central, the 'PsycINFO' database and CAS,

\section{Dovepress}

and is the official journal of The International Neuropsychiatric Association (INA). The manuscript management system is completely online and includes a very quick and fair peer-review system, which is all easy to use. Visit http://www.dovepress.com/testimonials.php to read real quotes from published authors. 